\title{
Proposal for Medical Data Transmission in Healthcare Systems
}

\author{
Type of article: Conference abstract
}

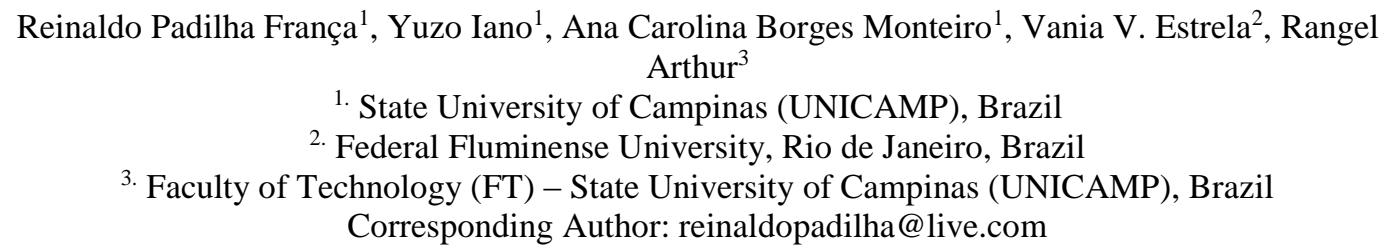

Abstract:

Background: Information systems used in hospitals are slow and consume a lot of system memory, facilitating crashes, impacting patients seeking consultation face long waiting periods by a medical specialist; Still considering that exchange patient data and medical consultations in system interconnected between hospitals, for scheduling of consultations may become even more latent.

Methods: Aiming to solve such problems, the present study implements modeling with discrete-event technology applied to a healthcare system, modulating the signal transmitted with the DQPSK format, through the simulation environment, the Simulink of the MATLAB software, improving the transmission of data, through a pre-coding process of bits adopting discrete events in the signal before modulation.

Results: This study aims to increase the information capacity for healthcare systems, bringing a new approach for signal transmission, undertaken in the discrete domain employing the discrete entities in the bit generation process, this use being the differential applied on the bit itself, in the physical layer, showing better computational performance regarding memory utilization related to compression of information, showing an improvement of $101.52 \%$.

Conclusion: The proposal developed has the properties of improving the capacity of hospital services and can increase the performance of the communication between all medical devices, this positive impact is the result that the data stream will consume fewer communication resources.

Keywords: eHealth, Telehealth, Telecommunication, Telemedicine, mHealth.

\section{Conflict of interest statement}

This article is a conference abstract presented at the International Congress on Health Sciences and Medical Technologies, Tlemcen Algeria 5-7 December 2019, ICHSMT'19.

\section{Authors' biography}

No Biography.

\section{References}

No references 